\title{
Reparación y reconciliación en el sistema normativo Wayuu ${ }^{1}$
}

\section{Reparation and reconciliation in the wayuu normative system}

Recibido: 16 de octubre de 2015 - Revisado: 23 de marzo de 2016 - Aceptado: 28 de abril de 2016.

\section{Nicolás Polo Figueroa ${ }^{2}$}

\section{Resumen}

En este artículo se explica en qué consiste la reparación y la reconciliación en el sistema normativo wayuu. El propósito es visibilizar una práctica de aplicación de justicia que busca restablecer la armonía entre los clanes de esta etnia (localizada en la península de la Guajira, Colombia), cuando esta se rompe por la agresión de uno a otro. Para su logro se muestra cuál es la concepción de impunidad para los wayuu, los efectos de la reparación en la sociedad y cómo se llega a la reconciliación. En general, se pretende demostrar la hipótesis de la investigación de cómo el sistema de normas está sustentado en la cultura de este pueblo.

\section{Palabras clave}

Jurisdicción especial wayuu, solución de problemas, palabrero, infractor, responsabilidad clanil, conflictos entre wayuus.

\begin{abstract}
This article explains what constitutes reparation and reconciliation in the wayuu normative system. The purpose is to make visible a practice of justice application that seeks to restore harmony between the clans of this ethnic group (located in the peninsula of Guajira, Colombia), when it is broken by aggression from one to another. To achieve this goal the conception of impunity for the wayuu is shown. As well as the effects of reparation in society, and how reconciliation is achieved. In general, the article tries to demonstrate the hypothesis of the investigation of how the system of norms is sustained in the culture of this people.
\end{abstract}

\section{Keywords}

Special wayuu jurisdiction, problem solving, babbler, offender, clanil responsibility, conflicts between wayuus.

\footnotetext{
1 Artículo resultado del proyecto de investigación: "El sustento cultural y ancestral del sistema normativo wayuu" adscrita al Grupo de Investigación Joaquín Aarón Manjarrés de la Escuela de Derecho de la Universidad Sergio Arboleda, Santa Marta, Colombia.

2 Doctor en filosofía de la Universidad de Iowa, Estados Unidos. Docente y Coordinador de la Universidad Nacional de Colombia. Investigador de la Universidad Sergio Arboleda, Santa Marta, Colombia. Director de la revista Verbum de la misma Universidad. Correo electrónico: nicolasignaciopolofigueroa145@gmail.com Para citar este artículo use: Polo, N. (2016). Reparación y reconciliación en el sistema normativo wayuu. Civilizar Ciencias Sociales y Humanas, 16(31), 41-48.
} 
Comienza la impunidad cuando una persona va a la cárcel Andrónico Urbay Ipuana

\section{Introducción}

El epígrafe del pütchipü’üi (palabrero) Andrónico Urbay, coordinador de la Junta Mayor de Palabreros, entidad responsable de llevar a cabo el Plan Especial de Salvaguardia (PES) del sistema normativo wayuu aplicado por el palabrero, aprobado por resolución 2733 del Ministerio de Cultura, evidencia de manera contundente cuál es la concepción de quienes tienen a su cargo la administración de la justicia especial wayuu. Una justicia en que los problemas de la etnia se resuelven mediante la conciliación, reparación, compensación y reconciliación ${ }^{1}$. Cuatro prácticas que están en el centro de la concepción de resolución de problemas entre los wayuus.

El objeto de este artículo es visibilizar cómo funciona dicho sistema en este aspecto, cuál es su concepto de impunidad, cuáles son los efectos de la reparación en la sociedad y cómo se llega a la reconciliación. De paso, sugerir este proceso de resolución de conflictos como alternativa a muchos de los problemas que se presentan en el país cuya única solución pareciera ser la cárcel.

Las informaciones que contiene este documento son producto de largas conversaciones con los palabreros Andrónico Urbay Ipuana, José Manuel Pana Epiayu y Orangel Gouriyu y con el gestor cultural y coordinador del PES, Guillermo Ojeda Jayariyu. Así mismo, recoge las propias vivencias del investigador como invitado a actos de reparación. Es, por tanto, el fruto de haber abrevado en fuentes directas de información. Por otra parte, el ajuste y fidelidad del contenido de este artículo a la realidad del sistema normativo de los wayuus fueron revisados por estas mismas fuentes y correlacionados con fuentes primarias (Alarcón, 2009; Colmenares, 2006; Guerra, 2002, 2006; Guzmán, 1997; Martínez,
\& Hernández, 2005; Mejía, 2011; Morales, 2004; Ojeda, 2013a, 2013b; Perafán, 1995; Pico, 2010; Pocaterra, 2006; Pontón, 2014; Santamaría, 2010; Segovia, Martínez, Malaguera, \& Ferreira, 2009, entre otros).

\section{Reparación de conflictos entre wayuus}

Las informaciones orales y la tradición nos cuentan que el wayuu desea vivir en paz; esta nos relata que las abuelas, las madres y las tías acostumbran a decir:

[...] todo problema debe arreglarse porque es muy bueno andar con seguridad y tranquilidad por los caminos. Es bueno no tener problemas con nadie; puedes andar libremente, dormir en el monte, andar detrás de los animales, cazar, pastorear, trabajar en cualquier cosa sin temor de tener enemigos (Urbay, 2014, p. 10).

Para lograr este estado de paz, convivencia y ausencia de problemas el wayuu debe reparar sus faltas, sean estas graves o simples ofensas de palabra: "alguien causó un daño, alguien calumnió, alguien escupió, alguien cacheteó, alguien robó, tiene que reparar el daño, esa ha sido la manera como nosotros milenariamente hemos resuelto todo entre los wayuu" (Urbay, 2014, citado en Potón, 2014). Perafán (1995) hace una enumeración y descripción de las conductas en que pueden incurrir los miembros de la etnia, sin excepción alguna. Precisamente este es uno de los temas centrales de la mesa de concertación entre la jurisdicción ordinaria y la especial wayuu, instalada el año pasado en el mes de noviembre en la ciudad de Riohacha, Colombia.

La reparación se hace mediante un pago. Este es proporcional a la falta cometida. Consiste en la entrega de ganado vacuno, ovino o caprino, tumas y collares. En la actualidad, ante la ausencia de estos semovientes y objetos, se está usando el dinero. Una verdadera pérdida, dado el valor simbólico de que gozaban las tumas y collares en esta cultura milenaria, centrada en la figura de la mujer. 
Tal mecanismo de indemnización es lo único que los wayuus consideran que restablece la armonía quebrantada por la agresión de un miembro de un clan contra otro. En este sentido, creen que el sistema de nuestra justicia ordinaria que castiga con cárcel a los crímenes cometidos conduce a la impunidad. A este respecto, Andrónico Urbay, en relación con lo adoptado por la justicia ordinaria en el caso de la masacre de Bahía Portete ${ }^{2}$, le contó a su entrevistadora Elizabeth Pontón (2014) lo siguiente:

Para nosotros comienza la impunidad cuando una persona va a la cárcel porque entonces no hay reparación. Aquí se necesita que la persona esté en plena libertad para que haya reparación. Lo que es interesante ahí identificar es a qué familia pertenece, para que puedan responder los familiares. [...] Aquí no hay una responsabilidad personal sino clanil (p. 42).

Esta indemnización o compensación que debe ser proporcional a la falta cometida, genera dos grandes efectos sobre la sociedad. Efectos que se sustentan en prácticas culturales y ancestrales; además, nos permite explicar ciertas conductas y actitudes que resultan "raras" (por decir lo menos) para los miembros de la cultura nacional.

Primero, establece lazos de solidaridad entre los miembros tanto de la familia del clan agredido como la del clan agresor. Como afirma el palabrero Urbay "aquí no hay responsabilidad personal sino clanil” (Pontón, 2014, p. 101). De manera que la reparación concierne a todos los familiares del clan agresor; una totalidad constituida por la primera autoridad del clan, el tío materno, sus sobrinos (los hijos de su hermana), los otros tíos y tías, el padre y sus hijos; incluso, a los amigos del clan; todos son responsables de reparar la afrenta infringida $y$ de una $u$ otra forma tienen que colaborar en la consecución del monto acordado para la reparación. A este respecto son significativas las palabras del Sr. Reverol Jayariyu en un acto de reparación al que se nos invitó: "tengo una responsabilidad con mi vida y de cumplir de acuerdo con los usos y costumbres de mi etnia" (Polo, 2015).

Por otra parte, esos lazos de solidaridad se observan entre los miembros del clan agredido al ver rota la armonía social. Aumenta en ellos el sentimiento de que merecen obtener una reparación so pena de convertirse en objeto de burla de los otros clanes; este se intensifica, a la par que crece en ellos la conciencia de los derechos que sus usos y costumbres les otorgan.

Segundo, la reparación tiene como objetivo la no repetición. Tanto el monto alto de la reparación como el carácter colectivo de esta son estímulos poderosos para que el infractor no ose reincidir. Martínez y Hernández (2005) consideran que se trata de una sanción moral, "se busca ante todo, es que, dada la experiencia que significa un pago de esta naturaleza, la falta no se vuelva a cometer y los familiares que pagan mantengan en alerta a sus parientes o ejerzan un control social" (p. 120).

Un propósito que el sistema de la justicia ordinaria del país está muy lejos de alcanzar mediante penas de cárcel; de hecho, estas no cumplen con la finalidad de resocializar al condenado para que no reincida. Analicemos brevemente estos dos efectos.

Suele afirmarse que los wayuus son oportunistas porque se aprovechan de los problemas entre los miembros de sus clanes para enriquecerse. Este, como otros tantos mitos sobre dicho pueblo, no deja de ser una afirmación que mide, con el rasero occidental, las prácticas de esta cultura ancestral, tan colombiana como la adoptada como propia en el territorio nacional. La reparación tiene un monto alto con el fin de que les duela a todos los miembros del clan agresor; que les pese desprenderse de los objetos y semovientes que han acumulado con esfuerzo y dedicación.

Por ejemplo, las mujeres del clan (madres y tías) pueden despojarse de los collares, 
muchos de los cuales provienen de sus ancestros: madres, abuelas y bisabuelas; e incluso, de actos de reparación. Por ende, si este desprendimiento de lo material duele se está ante la idea de la no repetición; del rechazo al acto cometido por haber roto la armonía social de la etnia.

Cuando una mujer wayuu tiene que despojarse de sus tumas y collares -sus objetos más preciados- para entregarlos a la familia agredida, este hecho se constituye en motivo de señalamiento para el miembro que cometió la falta. Como gran parte de la fortuna del clan respalda la palabra empeñada, al infractor solo le queda la opción de buscar el camino que sus mayores le trazan y, en fin, de resocializarse. A este respecto son dicientes las palabras de Urbay al narrar situaciones de esta naturaleza:

Después de resolver el problema, los familiares del agresor se reúnen y dicen "mire, por usted, yo perdí mi cadena, vea ahora quedo con el cuello limpio, sin collares; el toro que estaba ahí me tocó entregarlo. Entonces yo no quiero que siga usted buscando problema. Quiero que de ahora en adelante usted madure, se responsabilice y se dé cuenta que el problema..." es como para darle un castigo al clan ese (Urbay, 2014).

Así mismo, la naturaleza colectiva de la agresión es el mejor antídoto que tiene la cultura contra el carácter díscolo de algún miembro del clan. Según Pocaterra (2006) "cualquier orientación de la conducta del wayuu sea positiva o negativa- existe siempre un autor cuyos actos tienen repercusiones colectivas" (p. 102). De su falta no solo es responsable el infractor, lo es: la primera autoridad del clan, por haber fallado en la formación y educación de ese miembro; y la madre, por no haber estado pendiente de la conducta de su hijo. Por otra parte, la responsabilidad colectiva de una agresión implica la pérdida de la paz, la tranquilidad y la movilidad. Si el pago es alto es, como dice Urbay, "para darle un castigo al clan ese".
En relación con el valor que le atribuyen los pütchipü'üi a la reparación, en el encuentro de estos en el XXVIII Festival de la Cultura Wayuu celebrado en Uribia (Colombia) del 12 al 15 de junio del 2015, el pütchipü'üi Andrónico Urbay recalcó que una reparación alta, onerosa, tiene como propósito disciplinar a los miembros de los clanes; estos no pueden ir creando conflictos entre las familias porque saben que afectan a todos sus miembros.

De igual modo, el líder wayuu Germán Aguilar considera que la reparación tiene como base el respeto entre los miembros de los clanes. Es el respeto a la integridad del clan cuya principal virtud es responsabilizarse por los errores cometidos por sus familiares; es el respeto a su tío materno cuya labor educativa queda en tela de juicio con los errores cometidos por sus sobrinos; es el respeto a toda la comunidad, en suma, el respeto a sí mismo. Esta práctica de imponer compensaciones onerosas para que sirva de escarnio al grupo es compartida por todos los pütchipü'üi agrupados en el PES.

\section{Papel del pütchipü 'üi en la reparación}

La reparación tiene por objetivo sanar las heridas que produce la agresión, la ruptura del tejido social y el irrespeto a la sana convivencia entre los miembros de la etnia ${ }^{3}$. El gestor y mediador, al acuerdo al que llegan las dos partes, es el pütchipü 'üi o palabrero, mensajero de paz y paladín de la equidad y de la sana convivencia.

El palabrero como mensajero del clan agredido parte del principio de que este le ha dicho la verdad. No es de su competencia averiguar, investigar acerca de los hechos objeto del conflicto. Por eso, la pregunta obligada es ¿cuál es su petición? ¿Su petición se basa en un hecho verdadero? Su tarea comienza en el momento en que el clan agredido considera que él es la persona más adecuada, por la reputación adquirida en el ejercicio de sus funciones, para "llevar la palabra" al clan agresor. No obstante, 
antes que él los visite una persona relacionada con ambos clanes, el keral, les notifica que el palabrero tal les llevará la palabra. De manera que cuando este llega a la ranchería de la familia agresora, esta ya sabe de antemano el propósito de la visita del palabrero.

El mensaje que el palabrero lleva es una estimación del costo de la reparación que la familia agredida cree justo. No obstante, antes de presentar el objeto de su mensaje a los miembros del clan agresor, el palabrero busca distender los ánimos mediante la función fática del lenguaje: introducción de conversaciones, en muchos casos, baladíes, sobre el estado del clima, los últimos acontecimientos del pueblo, la salud de la familia y los animales; algunos suelen emplear apuntes humorísticos. Espera entonces que los anfitriones le pregunten por el motivo de su visita.

Entra en materia nombrando las personas que lo han enviado y recalcando el hecho de que es un emisario que transmite los deseos del clan agredido. Empero, usa su retórica para insistir en la necesidad de que las partes lleguen a un buen arreglo. Dicho el mensaje, la autoridad del clan le informa que ellos le harán saber al clan agredido cuándo se podrán reunir para darle una respuesta a la propuesta de reparación. En muchos casos, el lapso de tiempo para la respuesta puede ser uno o dos meses de acuerdo con las diligencias necesarias para obtener lo que consideran pueden conseguir con sus familiares que, como se dijo antes, son responsables solidarios del error cometido.

La actuación del palabrero conlleva siempre su sentido de equidad; fundada en que en cualquier arreglo debe siempre brillar la verdad y la justicia. Ahora bien, ante la contrapropuesta de la parte agresora, la agredida no siempre la acepta. En esta circunstancia el palabrero también debe servirse de su retórica en busca de que las partes lleguen a un consenso. Para ello usa argumentos similares a los esgrimidos con la parte agresora. Debe lograr una aproximación a la de la contrapropuesta y la lleva de nuevo a la contraparte, siempre en busca de que las diferencias se acorten y se marche en pro del acuerdo, de la solución conflictiva.

Uno de sus argumentos más persuasivos tiene como referente las venganzas que surgen porque las partes no pueden llegar a un acuerdo justo, a pesar de los buenos oficios del palabrero. Por eso, un buen pütchipü'üi debe estar informado de la vida de sus conciudadanos. $\mathrm{Si}$ consigue poner de acuerdo a las partes se establece una fecha para la compensación o indemnización.

Llegado el tiempo para el acto de compensación, todos los miembros de ambos clanes se reúnen en dos sitios no lejanos para que el pütchipü'üi pueda realizar su labor de mediador. Dos lugares acondicionados para que él pueda dirigirse cómodamente a las primeras autoridades de los clanes: el agresor y el agredido. Para él comienza un ir y venir entre los dos clanes, transmitiendo los deseos de ambas partes. Su labor entre los agresores es lograr una aproximación a las exigencias de la parte agredida sobre el monto de la reparación. No siempre es fácil la tarea.

En muchas ocasiones tiene que echar mano de argumentos persuasivos acerca de las ventajas y desventajas de un buen arreglo. Para señalar las primeras muestra cómo, en casos similares, las familias conviven en sana paz (la paz asociada a poder andar desprevenidos por los caminos); adquieren lazos familiares por matrimonios entre los miembros de los clanes en conflicto; estrechan la solidaridad entre los miembros del clan. Para enfatizar en las consecuencias negativas: no arreglar lleva a la mente de los asistentes las desazones, las guerras violentas, las venganzas ${ }^{4}$, las pérdidas de vidas humanas, lo más preciado del wayuu, que han ocurrido por no arreglar el problema. De ahí que el conocimiento de los conflictos entre familias sea parte esencial de los saberes que debe tener el pütchipü'üi. 
Ahora bien, no siempre la reparación se ejecuta en un solo acto. Cuando la compensación es alta las partes acuerdan el pago en varios plazos. El primer pago, conocido como Ishou'püna, garantiza la formalidad y el respeto del acuerdo; en los casos de homicidio, al padre se le reconoce un pago especial, la Ou'wiiraa, como forma de resarcir el derramamiento de sangre de su hijo. El valor principal de la agresión se le conoce como la Jutawaa. El pago final es la Jirawaa.

\section{La reconciliación en la cultura wayuu}

Al revisar los siete sentidos que Beristain (2005) le asigna al término reconciliación, ninguno le es aplicable a la práctica cultural de los wayuus. Para ellos, la reconciliación es el fruto de la reparación generada en el reconocimiento de los daños producidos con la agresión de un miembro de un clan contra otro. Una reparación que parte de la verdad de lo acontecido. Opera mucho después de que todo el proceso de compensación se ha agotado y las dos partes consideran que la ruptura del tejido social se sanó. La reconciliación se realiza de acuerdo con un ritual cultural.

El ritual consiste en la reunión de los dos clanes enfrentados: se juntan por primera vez, pues por costumbre se sugiere que los miembros de los clanes no tengan encuentros anteriores. En ningún momento de la reparación las partes han estado frente a frente; siempre han guardado distancia. El palabrero es el puente entre ellas. Es costumbre no socializar entre partes, sobre todo, en reuniones en que el alcohol está de por medio, no sea que se puedan atizar los ánimos.

Reunidos en una ranchería las autoridades de los clanes y los asistentes, toman tres pequeños tragos de licor; uno por uno, pero no más "no sea que se les suban a la cabeza". Ahí termina el rito y los miembros de los clanes se separan. Los efectos de este acto en que se relatan los consejos de las madres y abuelas, están descritos líneas arriba.

\section{A manera de conclusión}

Para los wayuus el sistema de penas de la justicia ordinaria del país lleva en sí el germen de la impunidad, no resocializa ni, mucho menos, conduce a la reconciliación. En cambio, en el sistema de la justicia wayuu, la víctima, no solo la personal sino la colectiva, recibe una compensación que permite urdir, en parte, el tejido social quebrantado. La resocialización del victimario se va gestando dentro del grupo clanil asaeteado por los reproches de los miembros del clan que perdieron sus objetos y posesiones. La reconciliación es la muestra de que la armonía ha conducido a las partes al estado inicial roto por la agresión de un miembro díscolo.

\section{Notas}

${ }^{1}$ La concepción que recoge este artículo es la tradicional de la etnia wayuu, una visión que los responsables de direccionar el PES (Ojeda, 2013a) están tratando que la justicia ordinaria respete en consonancia con el artículo 246 de la Constitución y el acuerdo PSAA-129614 de la Sala Administrativa del Consejo Superior de la Judicatura.

${ }^{2}$ Este es uno de los más sonados casos en que los paramilitares masacraron a trece mujeres de la etnia, en el 2004. Un acto en que José María Barrios Ipuana, un indígena wayuu, fue condenado a cuarenta años de cárcel a pesar de haber solicitado ser juzgado por la jurisdicción especial wayuu. Las consecuencias de esta solución, por fuera de la jurisdicción indígena, son hoy día objeto de discusión y revisión, por cuanto la jurisdicción ordinaria como en muchos otros casos en vez de solucionar los conflictos los empeora (véase Guzmán, 1997).

${ }^{3}$ Hay una discusión acerca del carácter de la labor del palabrero, ¿son árbitros, defensores legales, intermediarios, mediadores o simples conciliadores? Una discusión que se puede ampliar en las obras de Perafán (1995) y Guerra (2006). 
4 Sobre la práctica de la venganza, comúnmente asociada con la ley del talión, sostiene Guzmán (1997) "se debe resaltar el hecho de la violencia, [...] nunca es el primer recurso al cual se acude, sino por el contrario surge como guerra cuando se han agotado las demás instancias de la negociación, más concretamente el cobro" (p. 101).

\section{Referencias}

Acuerdo No. PSAA10-7543 de 2012. Por el cual se establecen los medidas de coordinación inter-jurisdiccional y de interlocución entre los Pueblos Indígenas y el Sistema Judicial Nacional. Sala Administrativa del Consejo Superior de la Judicatura, julio de 2012.

Alarcón, J. (2009). Palabra y poder. El 'pütchipü' y el cruce de fronteras culturales. Gazeta de Antropología, 25(1). Recuperado de http://www.ugr.es/ pwlac/ G25_05Johnny_Alarcon_Puentes.pdf

Beristain, C. (2005). Reconciliación: Desafios y experiencias. V Congreso Internacional de Museos por la Paz "GernikaLumo", España. Recuperado de http:// www.museumsforpeace.org/attachments/ article $/ 5 / 18 \% 20$ carlos $\% 20$ beristain $\% 20$ (8)\%20150-157.pdf

Colmenares, R. (2006). Sistema de justicia penal formal y el derecho consuetudinaria wayuu. Frónesis, 13(1), 57-69.

Constitución Política (1991). Congreso de la República de Colombia. Colombia.

Guerra, W. (2002). La disputa y la palabra. La ley en la sociedad wayúu. Bogotá: Ministerio de Cultura.

Guerra, W. (2006). Los conflictos interfamiliares wayúu. Frónesis, 13(1), 40-56.
Guzman, F. (1997). Venganzas de sangre entre los wayu frente al derecho penal colombiano. Revista de Universidad de los Andes, 9(1-2), 93-106.

Martínez, S., \& Hernández, A. (2005). Territorio $y$ ley en la sociedad wayuu. Riohacha: Fondo Mixto para la promoción de la Cultura y las Artes de la Guajira.

Mejía, P. (2011). Situación sociolingüistico del wayuunaiki: ranchería El pasito (Tesis de maestría). Bogotá: Universidad Nacional de Colombia.

Morales, E. (2004). Zenú, Emberá y Wayúu, tres culturas aborigenes. Bogotá: Fondo Nacional Universitario.

Ojeda, G. (2013a). Plan especial de Salvaguardia (PES) del Sistema Normativo Wayuu aplicado por el palabrero. Territorio Ancestral Wayuu: Mincultura. Gobernación de la Guajira, Alcaldía de Maicao, Junta Mayor de Autónoma de palabreros. Recuperado de http://www. mincultura.gov.co/prensa/noticias/Documents/Patrimonio/02-El\%20sistema $\% 20$ normativo $\% 20$ way $\% \mathrm{C} 3 \% \mathrm{BAu} \% 20$ aplicado $\% 20$ por $\% 20$ el $\% 20$ palabrero $\% 20$ Putchipu\%E2\%80\%99ui\%20-\%20PES. pdf

Ojeda, G. (2013b).Contexto Social y Lingüistico de la mujer Ouutsü. Autoridad Espiritual Wayuu. Territorio Ancestral Wayuu: Mincultura, Gobernación de la Guajira, Alcaldía de Maicao, Junta Mayor de Autónoma de palabreros.

Perafán, C. (1995). Sistemas jurídicos Páez, Kogi, wayuu y Tule. Bogotá: Colculutra.

Pico, F.A. (2010). Memoria, entre la jurisdicción indígena y el sistema jurídico wayuu. Universitas Estudiantes, 7, 11-32. 
Pocaterra, J. (2006). Principios culturales y lingüísticos que orientan las normativas del derecho consuetudinario wayúu. Frónesis, 13(1), 92-107.

Polo, N. (12 de julio de 2015). Grabación del acto de reparación del Sr. Reverol Jayariyu. Maicao.

Pontón, E. (2014). Crítica a las normas institucionales de reparación a víctimas indígenas. Caso de Análisis: El pueblo Wayúu (Tesis de pregrado). Universidad del Rosario, Bogotá. Recuperado de http:// repository.urosario.edu.co/bitstream/ handle/10336/8949/1018426851-2014. pdf? sequence $=4 \&$ isAllowed $=y$

Resolución 2733 de 2009. Por la cual se aprueba el Plan Especial de Salvaguardia del Sistema Normativo Wayuu aplicado por el Pütchipü'üi (Palabrero), declarado como Bien de Interés Cultural del Ámbito Nacional e incluido en la Lista Representativa de Patrimonio Cultural Inmaterial. Ministerio de Cultura, diciembre de 2009.
Santamaría, C. (2010). Narraciones jurídicas y conflictos tribales en la alta y Media Guajira: el proceso de reconocimiento de los derechos territoriales de los indígenas wayúu de 1960 a 1990 (Tesis de pregrado). Universidad Javeriana, Bogotá. Recuperado de http://javeriana. edu.co/biblos/tesis/csociales/tesis159.pdf

Salcedo-Ramos, A. (2013). La palabra de Juan Sierra, palabrero wayúu- Entrevista de Andrés Salcedo.

Segovia, Y., Martínez, J., Malaguera, J., \& Ferreira, F. (2009). Estudio comparativo entre el sistema formal de justicia penal de Venezuela y el sistema vindicatorio de la sociedad wayuu. Recuperado de http:// www.cjp.ula.ve/cenipec/proyectos/con financiamiento/sociedadwayuu_cdcht.pdf

Urbay, A. (2014). Entrevista no grabada realizada por Nicolás Polo. Cuaderno No.2 (pp.1-30). Maicao. 